\title{
Pushing Surface X-ray Probes Toward Mesoscale and Ultrafast Transient
}

\section{Hua Zhou}

Advanced Photon Source, Argonne National Laboratory, 9700 South Cass Ave, Lemont, IL 60439

hzhou@anl.gov

Ubiquitous in a wide range of nature processes and technologies, a subtle modification (e.g. structurally, chemically, or electronically) near surface can have a decisive effect on properties of the collective as well as each individual. Surface X-ray scattering from modern synchrotron sources integrated with phase retrieval methods, in particular crystal truncation rod (CTR) technique, provides a very powerful toolkit to decipher the surface mystery. This is essential to our ability to provide a quantitative and realistic description of the surface boundaries by which to engineer properties of functional surfaces using atomic structure-driven design principles in a reliable and controlled manner. However, its application to probing surface structural properties remains limited to the macroscopic dimension (e.g. $\mathrm{mm}$ single crystal/thin film), flat surface (e.g. zero surface curvature), and static systems.

High-brilliance, high flux synchrotron source capable of routinely realizing nanobeam below 100 $\mathrm{nm}$ and the state-of-art free-electron laser source delivering femtosecond $X$-ray pulses have make surface X-ray nanodiffraction for mesoscale surface systems and ultrafast surface X-ray scattering for evolving surface dynamics become practical and user-accessible. In this talk, we will firstly introduce the frontier development of surface X-ray nanodiffraction and ultrafast surface X-ray scattering measurements at both APS and LCLS facilities, and then demonstrate most recent intriguing practices on investigating 2D atomic thin crystal and Lego-style 2D heterstructures by utilizing the two techniques pushing spatiotemporal limits. In mesoscale regime, surface nanodiffraction can help to map out the complete specular CTR of a high quality graphene-hexagon BN heterostructure, revealing subtle variation of interfacial van-der-waals bonding between exfoliated and CVD grown 2D thin crystals. Moreover, surface nanodiffraction allowed for precise determining in-plane lattice expansion of miniature $\mathrm{MoS}_{2} 2 \mathrm{D}$ flakes grown on highly curved glass spheres, which provides an excitingly new approach to effectively manipulate electronic band valley structures [1]. In ultrafast transient domain, we have accomplished femtosecond surface $\mathrm{X}$-ray diffraction in combination with model-refinement calculations to quantify the ultrafast structural dynamics of monolayer $\mathrm{WSe}_{2}$ crystals supported on a substrate upon optical laser excitation [2]. It was illuminated that absorbed optical photon energy is preferably coupled to the in-plane lattice vibrations within one picosecond whereas the out-of-plane lattice vibration amplitude remains unchanged during the first ten picoseconds. The model-assisted fitting suggests an asymmetric intra-layer spacing change upon excitation, highlighting the distinct structural dynamics of monolayer crystals from their bulk counterparts. In summary, the demonstrated surface X-ray nanodiffraction and ultrafast surface X-ray scattering will bring about significant opportunities for us to explore emergent phenomena and develop novel functionalities at and across the surface and interface of a wide range of lowdimensional material systems.

\section{References}

[1] Zeng, M. Q. et al. (2019). Submitted to Nature Materials.

[2] Tung, I-C., et al. (2019). Nature Physics (DOI: 10.1038/s41566-019-0387-5). 